\section{Isolation and biological characterization of chicken amnion epithelial cells}

\author{
Y. Gao, ${ }^{1}$ Y. Pu, ${ }^{1}$ D. Wang, ${ }^{2}$ W. Zhang, ${ }^{1}$ \\ W. Guan, ${ }^{1}$ Y. Ma ${ }^{1}$ \\ 'Institute of Animal Sciences, Chinese \\ Academy of Agricultural Sciences, \\ Beijing; \\ 2Department of Pathology, Chinese PLA \\ General Hospital, Beijing, China
}

\begin{abstract}
Amniotic epithelial cells (AECs) express Oct4, Nanog and Sox-2, which are necessary for maintaining the undifferentiated state of pluripotent stem cells. AECs additionally express CK19, which is a specific marker of epithelial cells, both in vivo and in vitro. In this research, we investigated the biological characteristics and potential for cell therapy of AECs from 6-day-old chicken embryos. We induced the AECs to differentiate into pancreatic islet-like cells (endoderm), adipocytes and osteoblasts (mesoderm) and neural-like cells (ectoderm), and used immunofluorescence and RT-PCR to detect the expression of AECs specific markers. To assess the differentiation capacity of AECs, passage 3 cells were induced to differentiate into adipocytes, osteoblasts, pancreatic islet-like cells and neural-like cells. The AEC markers, 0ct-4, Nanog, Sox-2 and CK19, were all positively expressed. Cloning efficiency decreased with increasing passage number. Passage 3 AECs were successfully induced to differentiate into pancreatic islet-like cells, osteoblasts, adipocytes, and neural-like cells. These results suggested that AECs isolated from chicken embryos exhibited the characteristics of the multipotent stem cells. AECs may therefore be ideal candidates for cellular transplantation therapy and tissue engineering.
\end{abstract}

\section{Introduction}

The chick embryo membrane is comprised of four parts: amnion, yolk sac, chorion (serosa) and allantois. ${ }^{1}$ The amnion is a thin membrane-lined cavity filled with fluid that plays a crucial role in embryonic development by, among other things, cushioning the embryo during development and preventing adhesion of the developing embryo to maternal structures. The amniotic membrane consists of five layers: the epithelium, basement membrane, compact layer, fibroblastic layer and spongy layer. The amniotic epithelial cells (AECs) are derived from the simple epithelium and express type I cytoskeletal 19 (CK19). Furthermore, AECs are isolated and identified by expression of the markers Nanog, Oct-4 and Sox-2, which are necessary for maintaining the undifferentiated state of pluripotent stem cells. Thus, AECs share similar characteristics with embryonic stem cells (ESCs). However, unlike ESCs, AECs do not express telomerase reverse transcriptase (TERT), and therefore do not form teratomas following transplantation into severe combined immunodeficiency mice testes. ${ }^{2}$ Amnion is a highly abundant and easily accessible tissue that may potentially be an important source of transplantable stem cells.

Until now, the vast majority of experimental materials have been obtained from human, mouse, rabbit and other mammals, ${ }^{3}$ rather than from poultry. The chicken is another model animal that provides an abundance of amnion tissues. In the present study, AECs isolated from 6-day-old embryonic chicken amnion tissues were cultured in vitro and identified by expression of specific surface markers, and then tested for their abilities to self-renew and differentiate. Our results may provide novel insights into the in vitro culture and characterization of AECs, and contribute to tissue reconstruction in avian species.

\section{Materials and Methods}

\section{Materials}

The materials used in this study were: DMEM/F12, fetal bovine serum (FBS, Gibco, USA); all-trans retinoic acid, dithizone (DTZ), dexamethasone, $\beta$-glycerophosphate, isobutyl methylxanthine (IBMX), vitamin $\mathrm{C}$ and insulin (Sigma-Aldrich, St. Louis, M0, USA); bFGF, EGF, and fibroblast growth factor-4 (FGF4) (Peprotech, Rocky Hill, TX, USA), rabbit antibody 0ct 4, rabbit antibody CK19, rabbit antibody Sox-2 (MBL, Nagoya, Japan), rabbit antibody neuron specific enolase (NSE), rabbit antibody Nestin, rabbit antibody neurofilament (NF) (Bioss, Beijing, China); mouse antibody glial fibrillary acidic protein (GFAP; Abcam, Cambridge, MA, USA), fluoroisothiocyanate (FITC) conjugated goat anti mouse secondary antibody IgG, FITC conjugated goat anti rabbit secondary antibody IgG (Zhongshan Golden Bridge, Beijing, China).

\section{In vivo immunolocalization of CK19 in tissue}

Six-day-old chicken (Gallus gallus)
Correspondence: Weijun Guan and Yuehui Ma, Institute of Animal Sciences, Chinese Academy of Agricultural Sciences, 2 Yuanmingyuan West Rd, 100193 Beijing, China.

Tel. +86.10.62815992 - Fax: +86.10 .62813463 .

E-mail:weijunguan301@gmail.com and

yuehui_ma@hotmail.com

Key words: chicken, AECs, biological research, differentiation capacity.

Acknowledgements: this research was supported by the Ministry of Agriculture of China for Transgenic Research Program (Nos. 2011ZX08009-003-006 and 2011ZX08012-002-06), the project of National Infrastructure of Animal Germplasm Resources (year 2012) and the central level, scientific research institutes for R\&D special fund business (2011cj-9, 2012zl072).

Contributions: YG and YP contributed equally to this work.

Received for publication: 4 January 2012. Accepted for publication: 26 April 2012.

This work is licensed under a Creative Commons Attribution NonCommercial 3.0 License (CC BYNC 3.0).

(C) Copyright Y. Gao et al., 2012

Licensee PAGEPress, Italy

European Journal of Histochemistry 2012; 56:e33 doi:10.4081/ejh.2012.e33

embryos were obtained under sterile conditions. The amnion layer was mechanically peeled off of the chicken embryo and cleaned by washing several times with PBS without calcium and magnesium. CK19 expression was detected by immunohistochemistry according to the method of Zhang. ${ }^{4}$

\section{Isolation of amniotic epithelial cells}

The amnion membrane was dissociated. The thin, almost transparent, amnion membrane, which contains AE cells and mesenchymal fibroblasts, was washed several times to remove blood. For isolation of AECs, amniotic membrane was trypsinized to release the AECs from the supporting connective tissue according to the method of Miki et al., ${ }^{5}$ but with a reduced trypsin digestion time. The membrane was incubated with $0.05 \%$ trypsin and $0.53 \mathrm{mM}$ EDTA for $1 \mathrm{~min}$ to dissociate debris from the surface of the membrane, followed by trypsinization for 5 min. Thus, most of the epithelial cells were removed from the amniotic membrane by trypsinization and the connective tissue layer remained intact. The viability of the AECs was determined by the trypan blue dye exclusion test and counting of the cells on a hemocytometer. 


\section{Cell culture and standard culture medium}

AECs were plated in six-well plates at a density of $5 \times 10^{4}$ cells per well in our standard culture medium, consisting of DMEM/F12 media supplemented with 10\% FBS, 2 mM L-glutamine (Gibco, Carslbad, CA, USA), 55 M 2-mercaptoethanol (Gibco), $20 \mathrm{ng} / \mathrm{mL}$ EGF and 10 ng/mL bFGF. The EGF and bFGF concentrations required to induce robust proliferation were defined in preliminary experiments. ${ }^{6}$ Upon reaching 70\%-80\% confluence, the cells were detached from the plates using $0.25 \%$ trypsin, which was quenched by addition of standard culture medium. The cell suspension was split into new plates at a ratio of 1:2 and incubated at $37^{\circ} \mathrm{C}$ in a $5 \% \mathrm{CO}_{2}$ atmosphere.

\section{Alkaline phosphatase staining}

Alkaline phosphatase (AKP) expression is an important determinant of ESCs in the undifferentiated state. AECs of passages 3,8 and 10 were stained for AKP expression according to Wang's method in order to determine whether the AECs were in the undifferentiated state.?

\section{Immunofluorescence}

Cells were fixed in $4 \%$ paraformaldehyde for 15 min and then washed three times in PBS (5 min each). The cells were permeabilized with $0.2 \%$ Triton X-100 for 20 min and washed three times in PBS (5 min each). Cells were blocked with $4 \%$ bovine serum albumin (BSA; Sigma) for $30 \mathrm{~min}$, and subsequently incubated in $3 \%$ BSA containing the following polyclonal antibodies: Oct-4 antibody (1:500), Sox-2 antibody (1:100), CK19 antibody (1:500), NSE antibody (1:100), and GFAP antibody (1:100), for $1 \mathrm{~h}$ at room temperature. Then, after washing three times in PBS (5 min each), the cells were incubated in PBS containing FITC-conjugated secondary antibody for $1 \mathrm{~h}$ at $37^{\circ} \mathrm{C}$ in the dark. After incubation, the cells were washed three times with PBS (5 min each) in the dark. Finally, nuclei were labeled by incubation with 4,6 diamidino-2-phenylindole (DAPI; Sigma). The cells were examined using a Nikon TE2000-E laser scanning confocal microscope.

\section{RT-PCR}

RNA was isolated from cells using the Trizol reagent (Invitrogen, Carslbad, CA, USA), cDNA was synthesized using a reverse transcription system (Takara, China), and the cDNA targets were amplified by PCR using the specific primer pairs shown in Table 1. The PCR products were visualized by electrophoresis on a $2 \%$ agarose gel.
Table 1. Primer sequences used in RT-PCR assay.

\begin{tabular}{|c|c|c|c|}
\hline Gene & Primer sequence & $\operatorname{Tm}\left({ }^{\circ} \mathrm{C}\right)$ & Fragment (bp) \\
\hline Sox-2 & $\begin{array}{l}\text { F 5' CAACGGAGGCTATGGGATG 3' } \\
\text { R 5' CGAATGAGACGAGGAGGTGA 3' }\end{array}$ & 56 & 282 \\
\hline Nanog & $\begin{array}{l}\text { F 5' CAGCAGACCTCTCCTTGACC 3' } \\
\text { R 5' TTCCTTGTCCCACTCTCACC 3' }\end{array}$ & 58 & 287 \\
\hline CK 19 & $\begin{array}{l}\text { F 5' GAAGAACCACGAGGAGGAAA 3' } \\
\text { R 5' TGTTCGGTATTGACGGCTAA 3' }\end{array}$ & 56.8 & 200 \\
\hline Collage type I & $\begin{array}{l}\text { F 5' AAGGATGGTCGCAATG 3' } \\
\text { R 5' GGTGGCTAAGTCTGAGGT 3' }\end{array}$ & 48.5 & 310 \\
\hline Osteopontin & $\begin{array}{l}\text { F 5' CAGAACAGCCGGACTTTC 3' } \\
\text { R 5' CTTGCTCGCCTTCACCAC 3' }\end{array}$ & 51 & 227 \\
\hline PPAR- $\gamma$ & $\begin{array}{l}\text { F 5' CTGTCTGCGATGGATGAT 3' } \\
\text { R 5' AATAGGGAGGAGAAGGAG 3' }\end{array}$ & 47.3 & 199 \\
\hline Lipoprotein lipase & $\begin{array}{l}\text { F 5' AGTGAAGTCAGGCGAAAC 3' } \\
\text { R 5' ACAAGGCACCACGATT 3' }\end{array}$ & 48.7 & 477 \\
\hline NF & $\begin{array}{l}\text { F 5' CCAGTCCGACCACAACAT 3' } \\
\text { R 5' TCCTGGTACTCCCTCAAAT 3' }\end{array}$ & 56 & 231 \\
\hline GFAP & $\begin{array}{l}\text { F 5'GAGGTGGATGCCAGCAAGCC 3' } \\
\text { R 5' GCCTCCAGGTCGCAGGTGAG 3' }\end{array}$ & 65 & 250 \\
\hline GAPDH & $\begin{array}{l}\text { F 5' TAAAGGCGAGATGGTGAAAG 3' } \\
\text { R5' ACGCTCCTGGAAGATAGTGAT 3' }\end{array}$ & 53 & 244 \\
\hline Insulin & $\begin{array}{l}\text { F 5' CTCTGTGGCTCCCACTTGGT 3' } \\
\text { R 5' GTATGTCTGTGCCCGCTTCC 3' }\end{array}$ & 60 & 267 \\
\hline Nestin & $\begin{array}{l}\text { F 5' CAACGAGCCTACATTGCTAA 3' } \\
\text { R 5' CTCATCTGGGAACTCACATTC 3' }\end{array}$ & 56 & 289 \\
\hline TERT & $\begin{array}{l}\text { F5'GTCAGAGCGAAGTCATCACAAGAAT3 } \\
\text { 'R 5'TGGCAAAACTCTGAAGTGACAAC3' }\end{array}$ & 55 & 117 \\
\hline
\end{tabular}
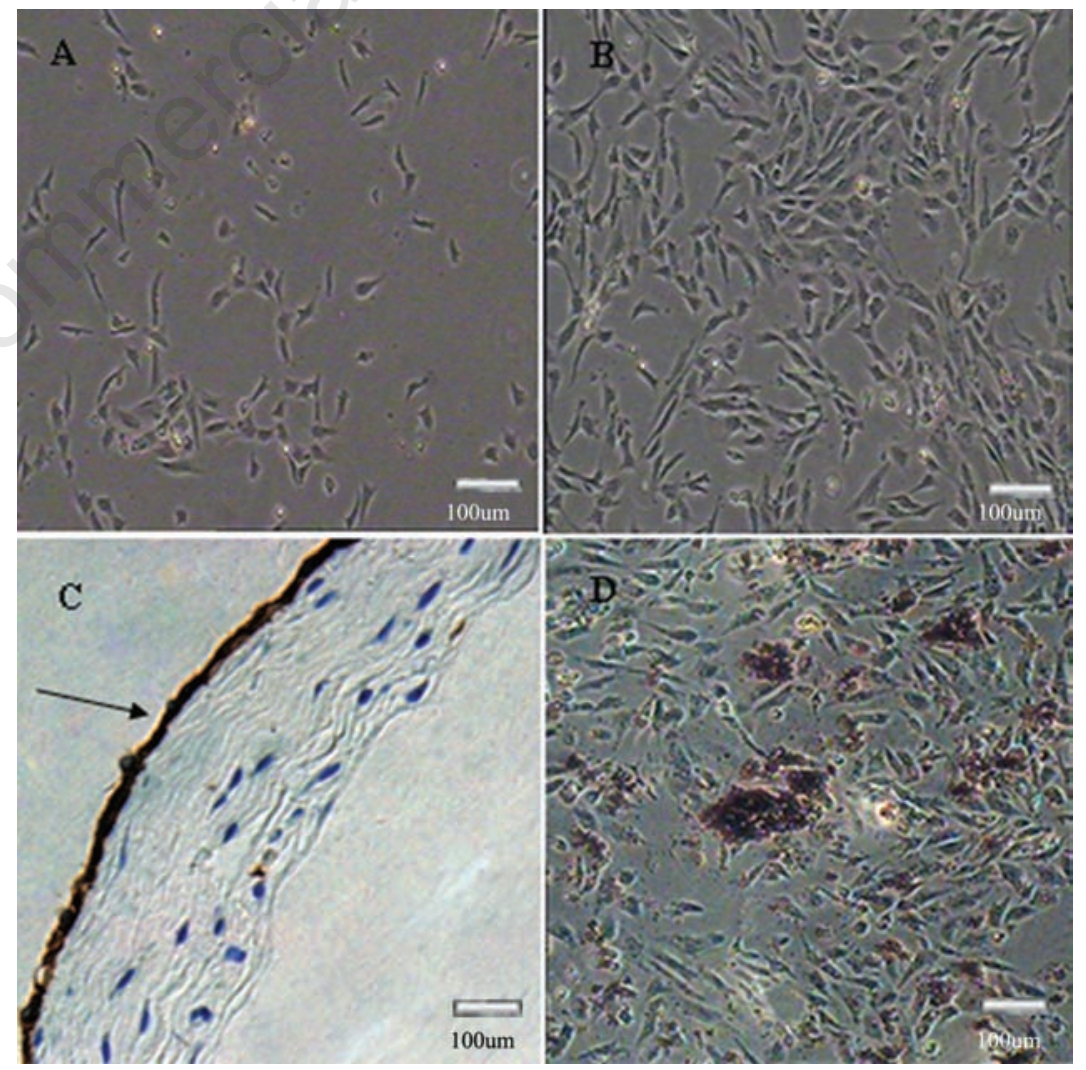

Figure 1. A) Primary cells after culture for $24 \mathrm{~h}$. B) AECs exhibited a round, oval or pyramidal shape in standard culture medium. C) Immunohistochemical detection of CK19 in amnion tissue; arrows indicate positive staining in the simple epithelial layer. D) AKP staining in AECs; the middle layer contains cells showing a strong positive reaction. A positive reaction is observed in the bottom of the adherent cells but the floating cells were negative. Scale bar: $100 \mu \mathrm{m}$. 


\section{Cloning efficiency}

Cells from passages 3,5 and 10 were seeded in $60-\mathrm{mm}$ plates at a density of $20 \mathrm{cell} / \mathrm{cm}^{2}$. After 2 weeks, the numbers of colony-forming units were counted and the cloning efficiencies were calculated as: colony forming unit number/starting cell number $\times 100 \%$.

\section{Differentiation of amniotic epithe- lial cells}

\section{Osteogenic differentiation of amniotic epithelial cells}

Cells were seeded in 24-well plates at a density of $1.0 \times 10^{4}$ cells/well. The cells were divided into two groups: the induced group and the control group. Upon reaching 50-60\% confluence, cells in the induced group were changed to osteogenic medium, consisting of DMEM/F12 medium supplemented with $10 \% \mathrm{FBS}, 0.1 \mathrm{mM}$ dexamethasone, $10 \mathrm{mM} \beta$-glycerophosphate and $50 \mathrm{mg} / \mathrm{L}$ vitamin $\mathrm{C}$. Meanwhile, cells in the control group were maintained in standard culture medium without any inducers of differentiation. The medium was changed every 2 days. Two weeks later, the cells' capacity for calcium node formation was detected by Alizarin Red staining, and osteoblast specific genes were further detected by RT-PCR.

\section{Adipogenic differentiation of amniotic epithelial cells}

Cells were seeded and divided into two groups as described above. Upon reaching 60 $70 \%$ confluence, cells in the induced group were incubated in adipogenic medium, consisting of DMEM/F12 medium supplemented with 10\% FBS, $1 \mathrm{mM}$ dexamethasone, $200 \mathrm{M}$ indomethacin, $0.5 \mathrm{mM}$ IBMX and $10 \mathrm{M}$ insulin. ${ }^{8}$ Cells in the control group were maintained in standard culture medium. After 7 days, intracellular lipid accumulation in the two groups was determined by staining with Oil Red 0. The RNA from the two groups was isolated for RT-PCR experiments.

\section{Neural-like differentiation of amniotic} epithelial cells

Cells were seeded and divided into two groups as described above. Neural-like cell differentiation was accomplished in DMEM/F12 medium supplemented with $10 \%$ FBS, $5 \times 10^{-5}$ $\mathrm{M}$ all-trans retinoic acid and $10 \mathrm{ng} / \mathrm{mL}$ FGF-4 (Peprotech). After 7 days, the cells were harvested and neural specific makers were detected by immunofluorescence and RT-PCR.

Pancreatic islet-like cell differentiation of amniotic epithelial cells

Cells were seeded and divided into two groups as described above. Pancreatic islet-like cell differentiation was accomplished in DMEM/F12 medium supplemented with $10 \%$ FBS and $10 \mathrm{mM}$ nicotinamide (Sigma). After 14 days, the two groups were stained with DTZ and the RNA was isolated for RT-PCR experiments.

\section{Results}

\section{Immunolocalization of CK19 in vivo}

The immunohistochemistry results showed that CK19 was strongly expressed on the internal surface of the amnion, but not in the others layers (Figure 1C).

\section{Isolation, culture and morphology of amniotic epithelial cells}

The isolated primary cells adhered to culture plates after $24 \mathrm{~h}$ (Figure 1A). The cells exhibited a round, oval or pyramidal shape with large nuclei (Figure 1B).

\section{Alkaline phosphatase staining of amniotic epithelial cells}

AKP staining showed that the cells located in the middle layer (cells were attached to the bottom of the adherent cells) displayed a strong positive reaction. The adherent cells at the bottom were also observed to be positive, but the floating cells were negative for AKP staining. The cytoplasm of the middle layer of cells observed on the membrane was also stained, suggesting that these cells can be maintained in the undifferentiated state after passaging (Figure 1D).

\section{Immunofluorescence}

Specific marker proteins for AECs were detected by immunofluorescence staining, and the results showed that 0ct-4 and Sox-2 were positively expressed. Positive expression of CK19 was also observed in the AECs. These results were similar to previous data on the characterization of AECs in vitro ${ }^{9}$ (Figure 2).

\section{RT-PCR analysis}

RT-PCR experiments showed that the AECs expressed the pluripotent stem cell markers, Nanog and Sox-2, and the specific marker of epithelial cells, CK19. TERT, which is expressed in ESCs, was also detected in AECs derived from chicken (Figure 3 ).

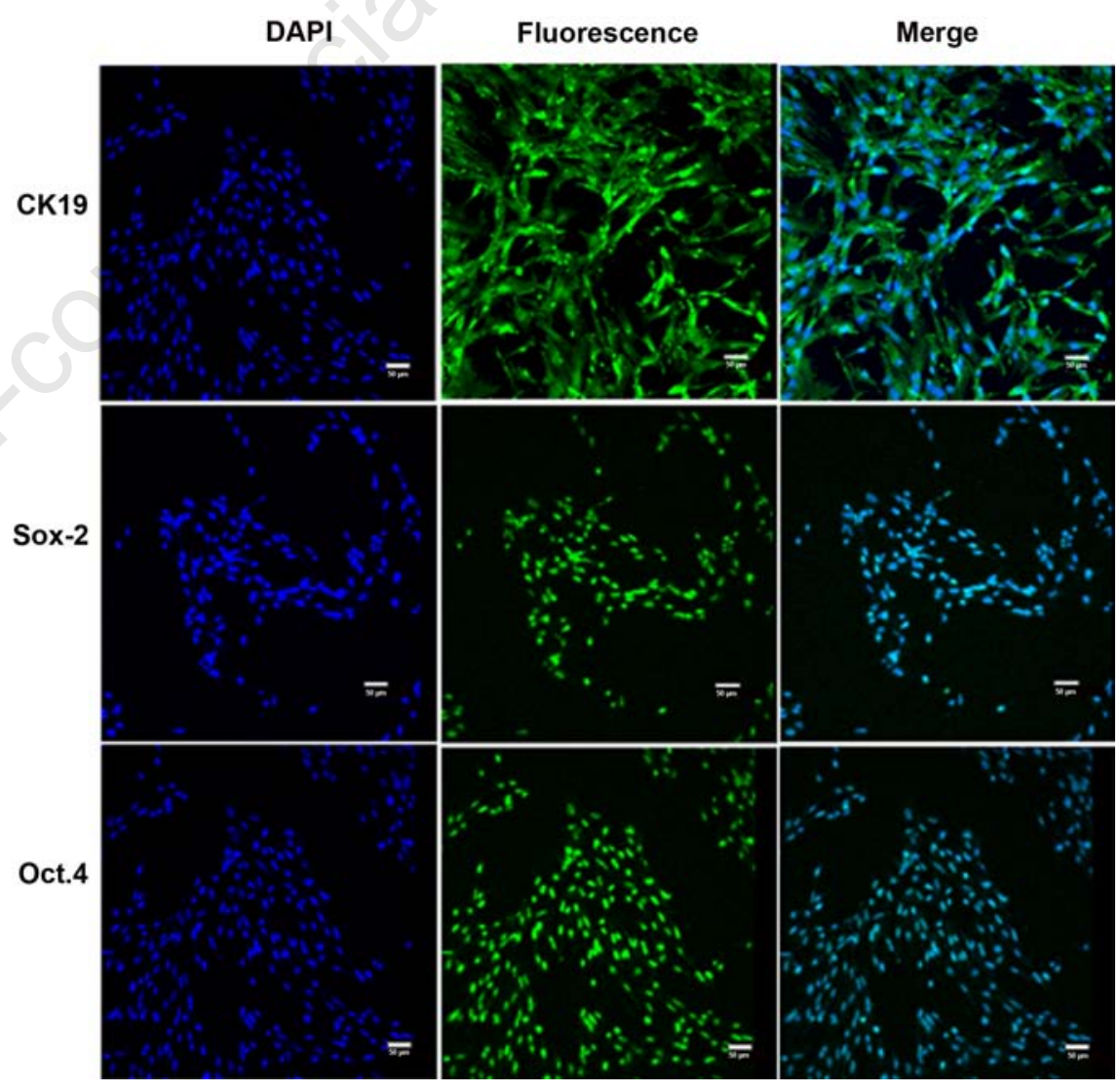

Figure 2. Immunolocalization of surface markers in AECs. Cell nuclei stained with DAPI are shown in the left panels. AECs with Oct-4 and Sox-2 nuclear staining, and CK19 staining in both the nucleus and cytoplasm, are shown in the middle panels (green). The merged images are shown in the right panels. Scale bar: $50 \mu \mathrm{m}$. 


\section{Cloning efficiency}

Colony formation was observed by microscopy after 14 days. The colony-forming efficiency rates were $31.6 \pm 0.24 \%, 29.2 \pm 0.19 \%$, and $15.6 \pm 0.32 \%$ for passage 3 , passage 5 , and passage 10 cells, respectively. These results demonstrated the self-renewal capacity of the cultured chicken AECs (Figure 4).

\section{Osteogenic differentiation of amni- otic epithelial cells}

After incubation in osteogenic medium for 14 days, the morphology of AECs changed markedly. Initially, the morphology of the cells changed from orbicular-ovate to tridimensional, and with time the cells aggregated and formed mineralized nodules. Alizarin Red staining identified a bright red positive region (Figure $5 \mathrm{~A}$ ). With continued time in culture in the presence of inducers of differentiation, the nodules increased in number and became larger in size. The morphology of the negative control cells cultured in complete medium was unaltered, and these cells were not stained by Alizarin Red (Figure 5B). Osteogenic differentiation of AECs was also determined by RT-PCR. Genes specific for osteoblasts, including collagen type I and osteopontin, were observed in the induced group but not in the control group (Figure 5C).

\section{Adipogenic differentiation of amni- otic epithelial cells}

Adipogenic differentiation of AECs was evidenced by positive Oil Red 0 staining. After incubation in adipogenic medium for 7 days, many lipid droplets were observed in the cells. With continued time in culture in the presence of inducers of differentiation, the number of droplets increased and small droplets aggregated to form larger ones (Figure 6A). The negative control cells cultured in complete medium for the duration of the culture process were not stained by Oil Red 0 .

Following induction with IBMX, insulin and dexamethasone, RT-PCR demonstrated the expression of the adipocyte specific genes, peroxisome proliferator-activated receptor (PPAR) $-\gamma$ and lipoprotein lipase (LPL). Expression of these genes was not observed in the control group (Figure 6C).

\section{Neural-like differentiation of amni- otic epithelial cells}

After incubation in neural differentiation medium, the cells exhibited elongated bodies and extended antennae. Cells with the morphologies of neurons and glial were observed after about 14 days (Figure 7 A,B). There were no obvious morphological changes in the control group. Moreover, Immunofluorescence demonstrated that the neuron cell marker

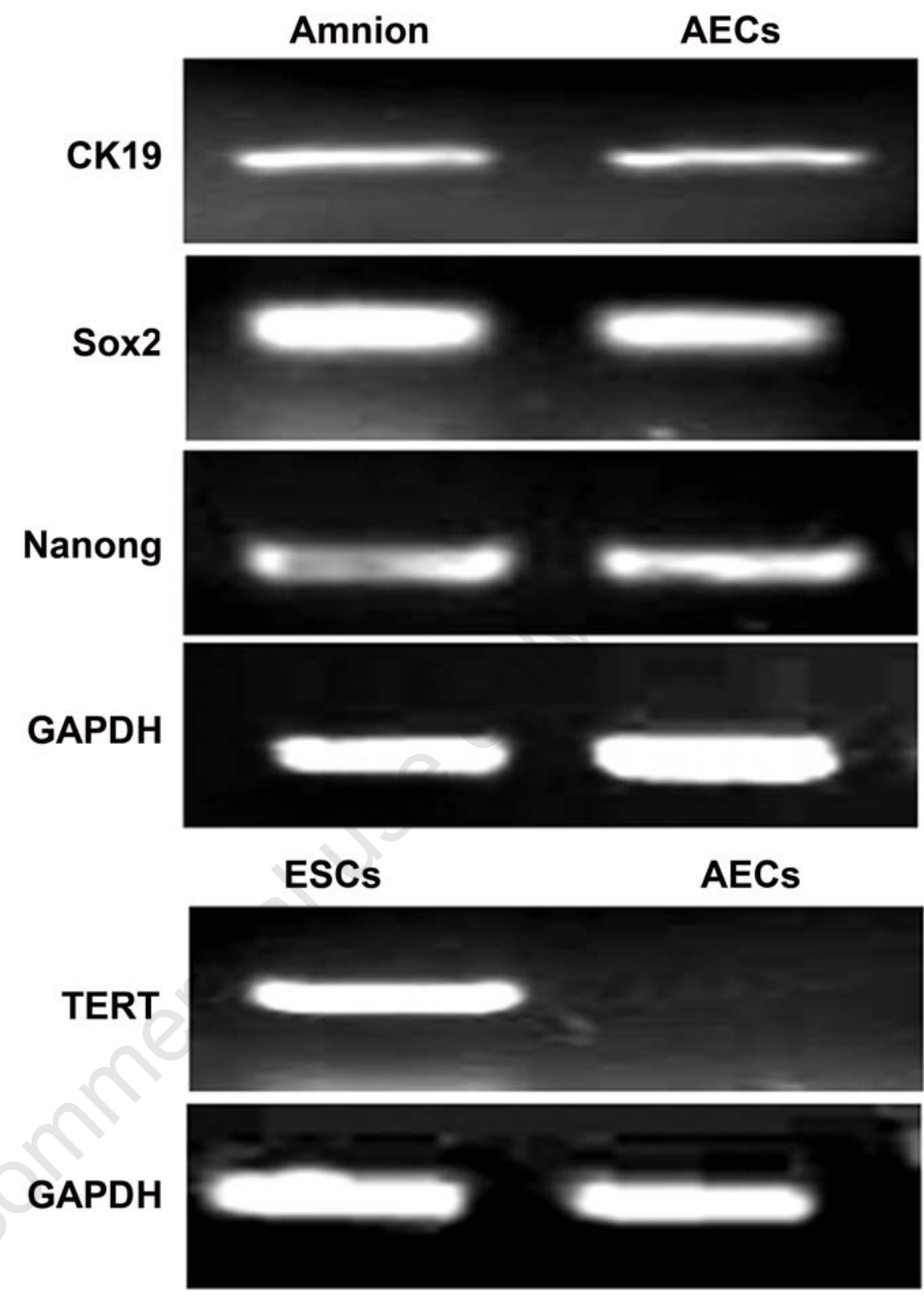

Figure 3. Reverse transcription-polymerase chain reaction analysis of AECs. Primers to stem cell-specific and cytokeratin genes were used.
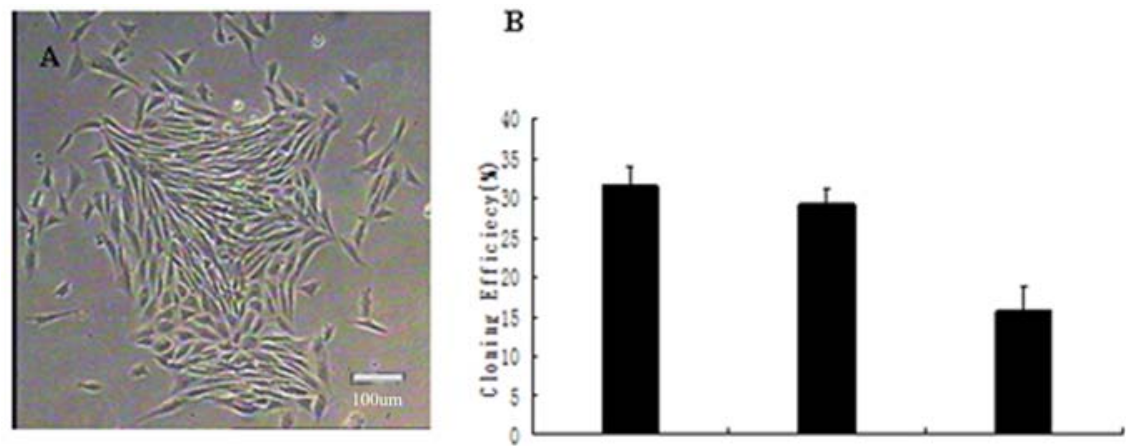

P马

75

710

Figure 4. Clon-genicity of AECs. A) Colonies with the morphology of AECs were cultured for 2 weeks (scale bar: $100 \mu \mathrm{m}$ ). B) Bar chart showing the cloning rates for different passages of AECs. 
(NSE) and the glial cell maker (GFAP) were expressed in cells in the induction group (Figure 8). RT-PCR demonstrated positive expression of Nestin, NF and GFAP genes in both the induction group and the non-induction group, but the relative expression levels in the induction group were significantly higher than in the control group (Figure 7D).

\section{Pancreatic islet-like cell differentia- tion of amniotic epithelial cells}

Pancreatic islet-like cell differentiation of AECs was evidenced by positive DTZ staining following incubation in induction medium for 14 days (Figure 9B). The negative control cells cultured in complete medium for the duration of the culture process were not stained by DTZ.

RT-PCR demonstrated that mRNA for insulin, a specific marker of pancreatic-like cells, was expressed in the induction group but not in the control group (Figure 9C).

\section{Discussion}

In this study, we have demonstrated that AECs express the stem cells markers, 0ct-4, Nanog and Sox-2, which are usually ascribed to self-renewal and pluripotency. ${ }^{10,11}$ Additionally, AECs exhibit clonal efficiency and can be differentiated in vitro into pancreatic islet-like cells (endodermal), osteoblasts and adipocytes (mesodermal), and neural-like cells (ectodermal). We also demonstrated that AECs stain positively for AKP. Taken together, these observations provide important and novel evidence for the multi-potentiality of AECs. We observed that AECs are clonogenic by the formation of large, flattened, undifferentiated colonies containing several hundred cells. Clonal efficiency, the ability of a single cell to form a colony, is a very important defining function that demonstrates the self-renewal potential of stem cells. ${ }^{12}$ In monolayer cultures of AECs, virtually $100 \%$ of the cells reacted with antibodies to CK19. These results confirmed their epithelial nature, and indicated that the cells did not arise from contaminating fibroblasts or mesenchymal cells.

TERT is an enzyme that adds DNA sequence repeats ("TTAGGG" in all vertebrates) to the 3 ' end of DNA strands in the telomere regions, which are found at the ends of eukaryotic chromosomes. ${ }^{13}$ Cancer cells and ESCs express this marker, which indicates the potential for telomerase activators to contribute to the development of tumors. ${ }^{14}$ Our finding that AECs did not express TERT suggests that AECs do not form tumors.

Dexamethasone combined with $\beta$-glycerophosphate and vitamin $\mathrm{C}$ was the most potent inducer of osteogenic differentiation. The induction process also depended on the time of application; the most effective induction time was 14 days after the initiation of culture. In the presence of $\beta$-glycerophosphate and vitamin $\mathrm{C}$, osteoblast cultures will spontaneously differentiate along a well character-
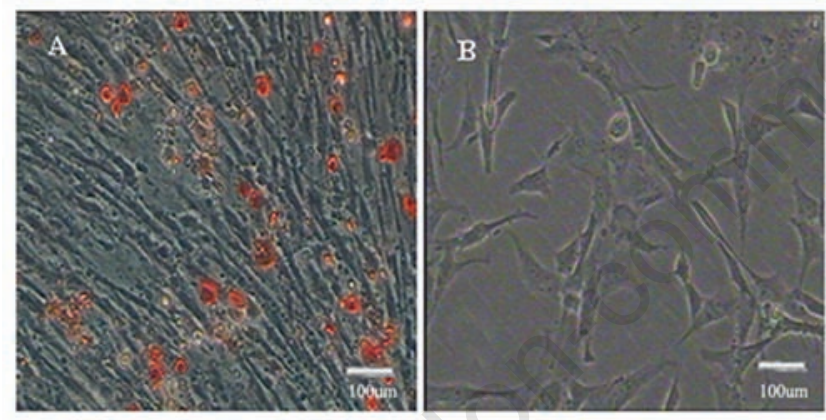

C

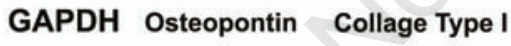

1

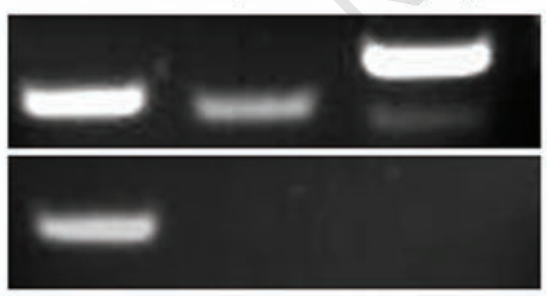

Figure 5. Osteogenic differentiation of AECs. A) After incubation in osteogenic medium for 10 days, the cells changed from orbicular-ovate to tridimensional in shape, and Alizarin Red staining was positive; the nodules increased in number and became larger with prolonged induction; about 14 days later, nodules were observed after Alizarin Red staining; cells cultured in complete medium did not change their morphology or stain with Alizarin Red. B) Control cells (Scale bar: $100 \mu \mathrm{m}$ ). C) RT-PCR revealed the expression of osteoblast specific genes, including osteopontin and collagen type $I$, in the induced group after incubation for 14 days; however, these genes were not expressed in control cells.

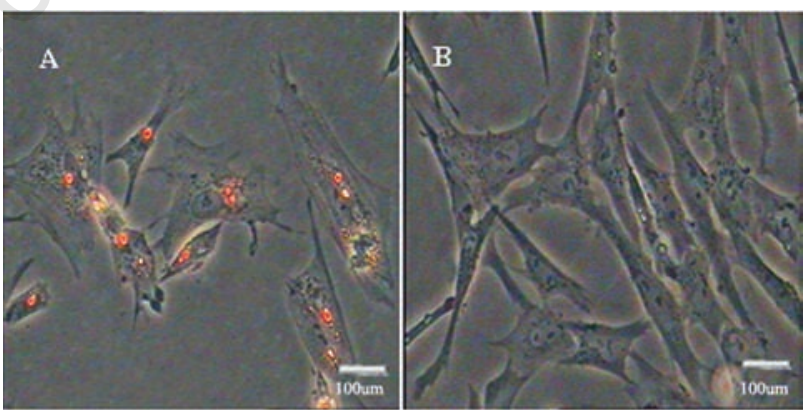

C

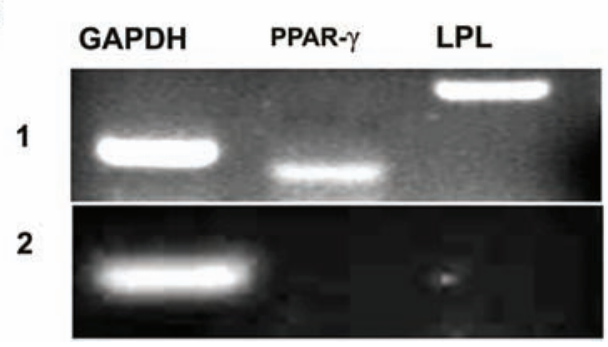

Figure 6. Adipogenic differentiation of AECs. A) After 1 week of induction, AECs changed from round, oval or pyramidal shape to oblate and contained many lipid droplets in their cytoplasm; the droplets increased in number and aggregated as induction progressed; cells cultured in complete medium throughout the culture process did not change their morphology and were not stained with Oil Red O. B) Control cells (scale bar: $100 \mu \mathrm{m})$. C) The expression of adipocyte-specific genes, including PPAR- $\gamma$ and LPL, was detected by RT-PCR in the induced group after incubation for 7 days, but these genes were not expressed in control cells. 
ized and ordered developmental pathway to form mineralized bone nodules. These nodules demonstrate the morphological and biochemical characteristics of stem cells and are useful for RT-PCR assay of osteoblast commitment and bone formation in vitro. ${ }^{15}$

In recent years, insulin, IBMX and indomethacin have been used to induce AECs to differentiate into adipocytes. ${ }^{16}$ This study showed that combined use of IBMX and dexamethasone is able to promote adipogenic differentiation of AECs by unknown mechanisms, probably because of the expression of PPAR- $\gamma 2$ and LPL. Oil red 0 is a fat-specific reagent, and was therefore used for fat droplet detection.

AECs are known to express certain neuronal and glial cell markers ${ }^{17}$ and to release neurotransmitters 3. Upon culturing AECs in neural differentiating medium, the cell numbers declined sharply. After 14 days, a small percentage of NF-positive neuronal cells with large central bodies and thin, elongated processes were observed. The majority of cells remaining after 14 days produced GFAP. These cells had large cell bodies and thin processes characteristic of astrocytes. These findings suggested the usefulness of AECs as an alternative source of cells for the treatment of neurological diseases.

Nicotinamide is a major factor required for the differentiation of AECs into pancreatic islet-like cells. RT-PCR for pancreatic islet cell genes was conducted on total RNA extracted from cells cultured in medium supplemented with $10 \mathrm{mM}$ nicotinamide. The mature hormone insulin was identified. Our experiments
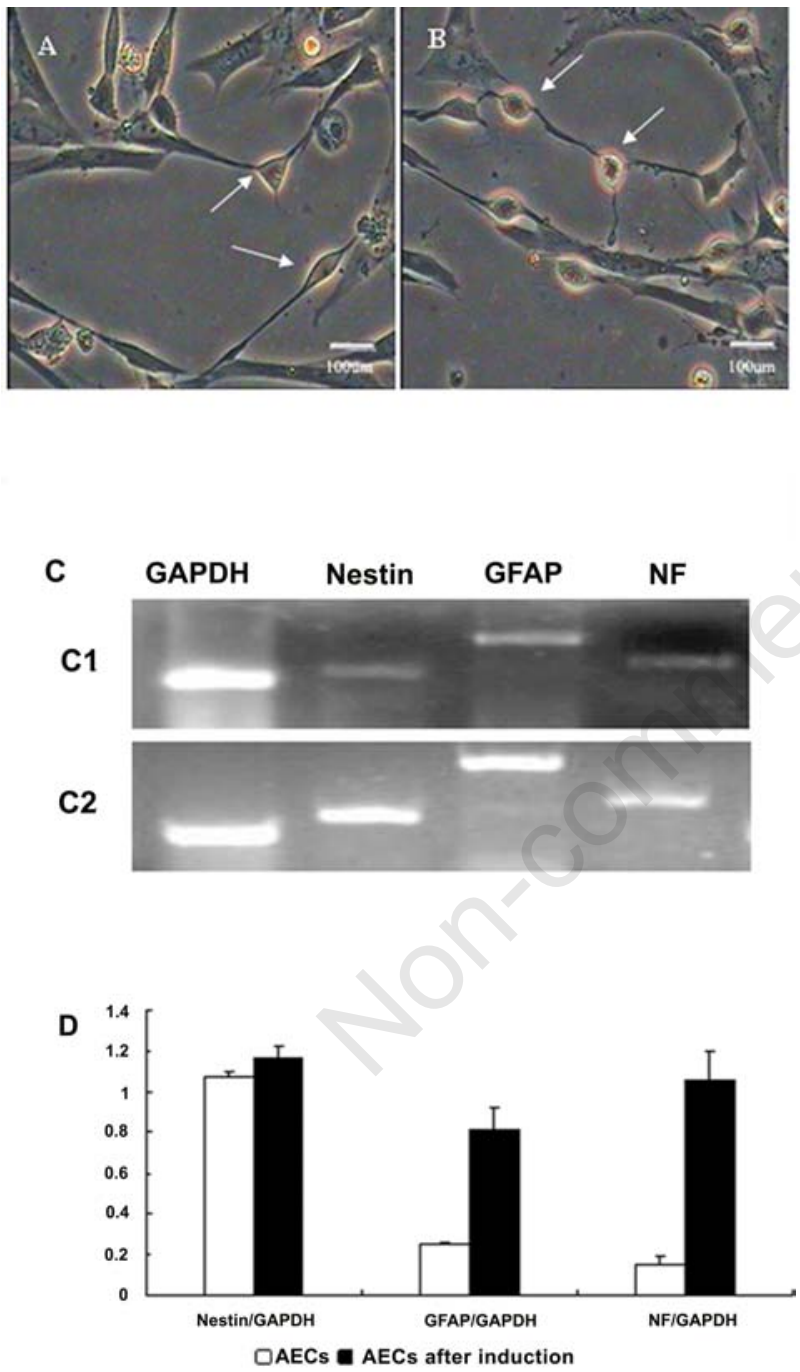

Figure 7. Neural differentiation of AECs. A) Neuron-like cells were observed after 2 weeks of induction, as indicated by the arrow. B) The arrow indicates glial cells (scale bar: $100 \mu \mathrm{m}) . \mathrm{C}$ ) The mRNAs for Nestin, GFAP and NF were increased in the induction group $(\mathbf{P}<0.05)$. D) RT-PCR revealed the expression of neural-like cell specific genes, including Nestin, GFAP and $\mathrm{NF}$, in both the induction group and the control group.
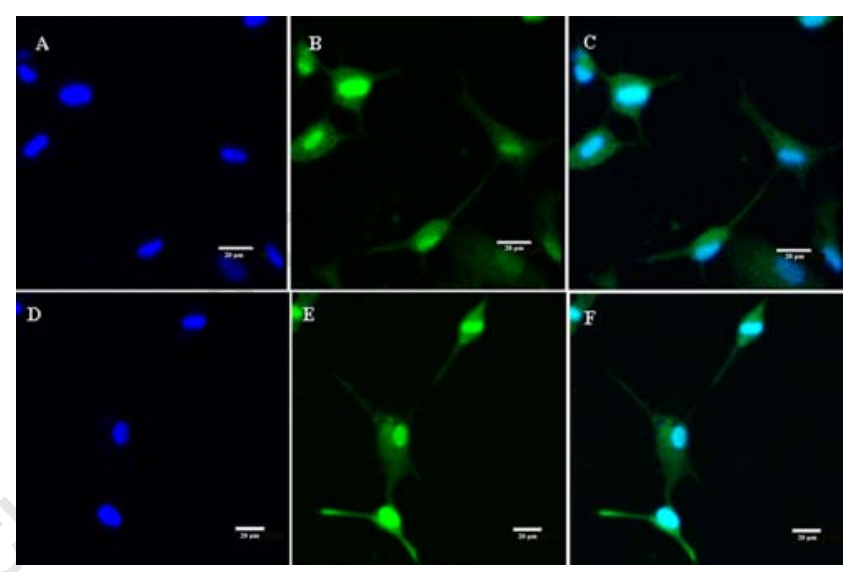

Figure 8. The neural-like cell markers, NSE and GFAP, were observed in the induction group by immunofluorescence. Scale bar: $20 \mu \mathrm{m}$.

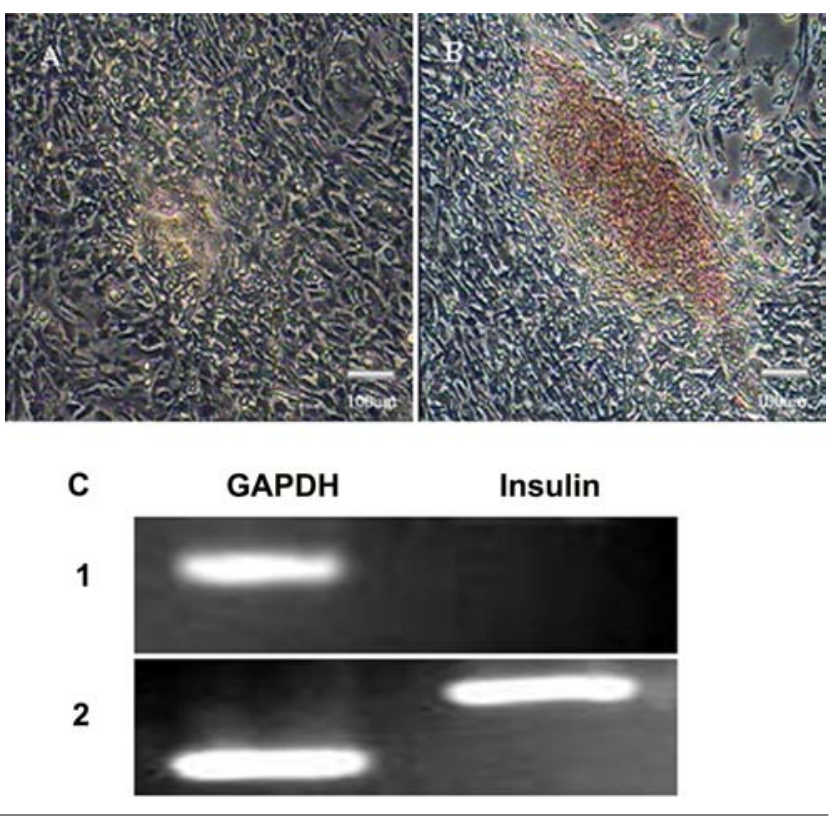

Figure 9. Pancreatic-like cell differentiation of AECs. A) After 2 weeks of induction, AECs aggregated to form a class of islet-like cell clusters. B) The AECs were induced after 2 weeks and were stained by DTZ, which is shown in brown (scale bar: $100 \mu \mathrm{m})$. C) Insulin mRNA was positively expressed in the induced cells. 
indicated that addition of nicotinamide to the culture medium of AECs both increases the total cell number and their differentiation into $\beta$-cells. The mechanism of this effect has been attributed to a simultaneous increase in the rate of DNA synthesis and the level of endocrine differentiation..$^{18}$ Nicotinamide also enhances the recovery from diabetes after $90 \%$ pancreatectomy both in rats 18 and dogs ${ }_{1}^{19}$ suggesting a stimulatory effect on islet regeneration.

In our study, we induced AECs isolated from chicken embryos to differentiate into osteoblasts, adipocytes, pancreatic-like cells and neural-like cells, and characterized them by the expression of genes for such cells. The results showed that different factors determined the differentiation pathway followed by the AECs. The autologous nature of these stem cells, together with their putative multi-potential and easy procurement, may make these cells an excellent choice for many future tissue engineering strategies and cell-based therapies. ${ }^{20}$ Although the multi-differentiation of AECs was successful in vitro, there are many drawbacks for utilizing these cells in tissue recovery in vivo, such as a higher decline rate and instable phenotype.

Therefore, further research into their utilization is needed.

\section{References}

1. Leng T, Miller JM, Bilbao KV, Palanker DV, Huie P, et al. The chick chorioallantoic membrane as a model tissue for surgical retinal research and simulation. Retina 2004;24:427-34.

2. Miki T, Lehmann T, Cai H, Stolz DB, Strom SC. Stem cell characteristics of amniotic epithelial cells. Stem Cells 2005:23:1549-59.

3. Elwan MA, Sakuragawa N. Evidence for synthesis and release of catecholamines by human amniotic epithelial cells. Neuroreport 1997;8:3435-8.

4. Zhang XJ, He JP, Wen XX, Zhao L. Immunolocalisation and expression of Smad2 and Smad4 proteins in dog testis during postnatal development. Andrologia 2011;43:254-60.

5. Miki T, Strom SC. Amnion-derived pluripotent/multipotent stem cells. Stem Cell Rev 2006;2:133-42.

6. Ilancheran S, Michalska A, Peh G, Wallace EM, Pera M, Manuelpillai U. Stem cells derived from human fetal membranes display multilineage differentiation potential. Biol Reprod 2007;77:577-88.

7. Wang Y, Hou L, Li C, Guan W, Chen L, Li X, et al. Isolation, culture and biological characteristics of primordial germ cells from Beijing fatty chicken. J Reprod Develop 2010;56:303-8.

8. Gong X, Hou L, Bai C, Jin D, He X, Guan W, et al. Isolation and biological characteristics of chicken adipose-derived progenitor cells. DNA Cell Bio 2011;30:453-60.

9. Chen YT, Li W, Hayashida Y, He H, Chen SY, Tseng DY, et al. Human amniotic epithelial cells as novel feeder layers for promoting ex vivo expansion of limbal epithelial progenitor cells. Stem Cells 2007;25:1995-2005.

10. Niwa H, Masui S, Chambers I, Smith AG, Miyazaki J. Phenotypic complementation establishes requirements for specific POU domain and generic transactivation function of 0ct-3/4 in embryonic stem cells. Mol Cell Biol 2002;22:1526-36.

11. Chambers I, Colby D, Robertson M, Nichols J, Lee S, Tweedie S, et al. Functional expression cloning of Nanog, a pluripoten- cy sustaining factor in embryonic stem cells. Cell 2003;113:643-55.

12. Chan RW, Schwab KE, Gargett CE. Clonogenicity of human endometrial epithelial and stromal cells. Biol Reprod 2004;70:1738-50.

13. Qiao F, Goodrich KJ, Cech TR. Engineering cis-telomerase RNAs that add telomeric repeats to themselves. P Natl Acad Sci USA 2010;107:4914-8.

14. Artandi SE, DePinho RA. Telomeres and telomerase in cancer. Carcinogenesis 2010;31:9-18.

15. Guan W, Wang Y, Hou L, Chen L, Li X, Yue $\mathrm{W}, \mathrm{Ma}$ Y. Derivation and characteristics of pluripotent embryonic germ cells in duck. Poult Sci 2010;89:312-7.

16. Sarugaser R, Lickorish D, Baksh D, Hosseini MM, Davies JE. Human umbilical cord perivascular (HUCPV) cells: a source of mesenchymal progenitors. Stem Cells 2005;23:220-9.

17. Sakuragawa N, Thangavel R, Mizuguchi M, Hirasawa M, Kamo I. Expression of markers for both neuronal and glial cells in human amniotic epithelial cells. Neurosci Lett 1996;209:9-12.

18. Otonkoski T, Beattie GM, Mally MI, Ricordi C, Hayek A. Nicotinamide is a potent inducer of endocrine differentiation in cultured human fetal pancreatic cells. J Clin Invest 1993;92:1459-66.

19. Sugiyama K, Yonemura Y, Okamoto $\mathrm{H}$. Effects of poly (ADP-ribose) synthetase inhibitor on B-cells of a canine pancreas after massive pancreatectomy. Int J Pancreatol 1991;8:85-95.

20. Zuk PA, Zhu M, Mizuno H, Huang J, Futrell JW, Katz AJ, et al. Multilineage cells from human adipose tissue: implications for cell-based therapies. Tissue Eng 2001; 7:211-28. 